\section{Ileal Metastases from Malignant Melanoma: Endoscopic Diagnosis}

Melanoma is increasing in frequency, and the most common metastatic disease found in the gastrointestinal tract is now metastatic melanoma (1). According to autopsy studies, the small intestine, and the ileum in particular, is the area most frequently involved $(58 \%)$, followed by the stomach $(26 \%)$ and the colon $(22 \%)(2)$.

The most common clinical presentations of small-intestine metastases are small-bowel obstruction by intussusception due to submucosal lesions (3), gastrointestinal bleeding, mainly chronic and occult, and diarrhea or constipation. The clinical presentation is very different from that of other metastatic tumors in the smallbowel, such as those from lung cancer, which often presents with acute perforation (4).

The diagnosis should be suspected in any patient with gastrointestinal symptoms and a history of cutaneous melanoma. The most reliable imaging test is $\mathrm{CT}$, and histopathological confirma-

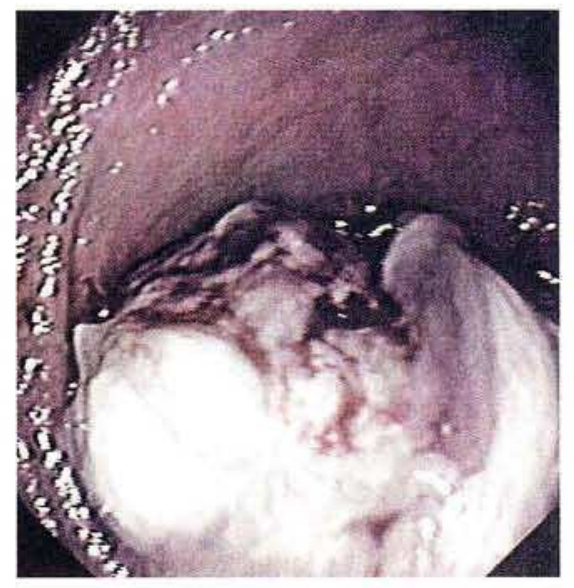

Figure 1: COLONO SCOPY: Endoscopic picture showing an exophytic ulcerated mass in the ileum.

tion is provided by surgical exploration ( $80 \%$ of patients), endoscopy, mainly the stomach $(15 \%)$, or cutaneous biopsy $(5 \%)$ (5). 
We report here the case of a 63 -year-old man who had undergone surgery for a cutaneous malignant melanoma located in the pectoral area five years previously. The patient was admitted to our hospital with severe iron-deficiency anemia (hematocrit 20\%). Diagnostic evaluation included a normal upper gastrointestinal endoscopy and ileocolonoscopy, during which an exophytic, ulcerated, polypoid mass was found in the terminal ileum (Figure 1). Endoscopic biopsies revealed malignant melanoma. The patient underwent surgery, during which two more ileal metastases were found. The main lesion was partially resected, and two bypasses were carried out in the intestine.

Intestinal resection for hemorrhage or obstruction provides symptomatic relief in $79-92 \%$ of patients, with a postoperative mortality rate of $5 \%$, but is mainly performed due to complications. Neither immunotherapy nor chemotherapy have been effective in prolonging the survival rates in these patients; the oneyear and five-year survival rates are $44 \%$ and $9-19 \%$, respectively (5).

F. Gomollón, S. Garcia, L. Gómez-Puch

Division of Gastroenterology, Miguel Servet Hospital, Zaragoza, Spain

\section{References}

1. Goldstein HR. Radiologic spectrum of melanoma metastatic to the gastrointestinal tract. Am J Roentgenol 1977; 129: 60512.

2. DasGupta TK, Brasfield RD. Metastatic melanoma of the gastrointestinal tract. Arch Surg 1964; 88: 969-73.

3. Lorigan JG, Du Brow RA. The computed tomographic appearances and clinical significance of intussusception in adults with malignant neoplasms. Br J Radiol 1990; 63; $257-$ 62.

4. McNeil PM, Wagman LD, Neifeld JP. Small bowel metastases from primary carcinoma of the lung. Cancer 1987; 59: 1486 9.

5. Capizzi PJ, Donohue JH. Metastatic melanoma of the gastrointestinal tract: a review of the literature. Compr Ther 1994; 20: $20-3$.

Corresponding Author

F. Gomollón, M. D.

Miguel Servet Hospital

Paseo Isabel La Católica 1-3

Zaragoza 50009

Spain 